\title{
Short Time Cycles of Purely Quantum Refrigerators
}

\author{
Tova Feldmann and Ronnie Kosloff \\ Institute of Chemistry the Hebrew University, \\ Jerusalem 91904, Israel
}

\begin{abstract}
Four stroke Otto refrigerator cycles with no classical analogue are studied. Extremely short cycle times with respect to the internal time scale of the working medium characterize these refrigerators. Therefore, these cycles are termed sudden. The sudden cycles are characterized by the stable limit cycle which is the invariant of the global cycle propagator. During their operation the states of the working medium possess significant coherence which is not erased in the equilibration segments due to the very short time allocated. This characteristic is reflected in a difference between the energy entropy and the Von Neumann entropy of the working medium. A classification scheme for sudden refrigerators is developed allowing simple approximations for the cooling power and coefficient of performance.
\end{abstract}

PACS numbers: 03.65.Yz,05.70.Ln, 07.20.Pe,05.30.-d 


\section{INTRODUCTION}

Magnetic cooling was initiated more than a half century ago [1]. The cooling agent paramagnetic salts was cooled both by adiabatic demagnetization [1] and by adiabatic magnetization [2] depending on the sign of the isothermal change of entropy as a function of the magnetic field. Currently, adiabatic demagnetization is an efficient technique for cooling detectors in space missions and also for home refrigerators without freon gas [3-6]. These devices are realizations of quantum refrigerators a subject which has been of growing interest in the last decade [7-24]. In this study we explore an extreme mode of operation of magnetic refrigerators where the short time allocation on the cycle segments leads to quantum characteristics.

A prototype of a magnetic four stroke refrigerator is investigated. The working medium of the refrigerator is a magnetic material modeled as an ensemble of two coupled spin systems [25, 26]. The working cycle is composed of four segments: two isomagnets, one magnetization and one demagnetization segment. The dynamics is generated by a completely positive map [27], which settles in a self repeating cycle - the limit cycle [28].

In the microscopic model two timescales emerge, the cycle time and the internal timescale determined by frequencies of the working medium. When the allocated time on each segment of the cycle is large compared to the internal timescale, the cycle is quasi-adiabatic. Under these circumstances the state of the working medium is close to an equilibrium or Gibbs state. These states are characterized by the expectation value of the energy. As a result the state $\hat{\boldsymbol{\rho}}$ is diagonal in the energy representation $[\hat{\boldsymbol{\rho}}, \hat{\mathbf{H}}]=0$.

When the cycle period becomes comparable to the internal timescale, the state of the system lag behind the change of the external parameters. The cause of this effect is that the external control Hamiltonian does not commute with the internal Hamiltonian. In this case, the states of the working fluid can not follow the instantaneous energy levels, therefore additional energy is stored in the working medium. This additional energy is accompanied by large off-diagonal elements of the state of the working medium $\hat{\boldsymbol{\rho}}$. The dissipation of this additional energy is the quantum analogue of friction [10].

A quantitative distinction can be made between two sets of cycles depending if the time spent on each segment is shorter or longer than the internal timescale This distinction is a boundary range between the two sets. The sudden cycles are characterized by segment 
times which are much shorter than the internal dynamical timescale. The regular cycles are those with segment times which are longer than the internal timescale. Our previous studies focused on regular cycles [22, 29]. Intermediate cycles exists where some segments have a short time allocation compared to the internal timescale and other segments have a long time allocation.

In the regular cycles the different segments can be characterized and studied individually. In [29] Fig. 4 is a representative example of those cycles where the different segments have many cycles, and can be treated almost independently, as opposed to the sudden cycles of the recent study. Typically, the states of the regular cycles are almost diagonal in the energy representation. This energy dominance is even more pronounced at the contact points between the cycle segments. The optimal cycles of the regular type are strictly diagonal in the energy representation on the contact points. These cycles are termed frictionless [19, 24, 29, 30].

The sudden cycles are characterized by large off-diagonal elements in the energy representation $[\hat{\boldsymbol{\rho}}, \hat{\mathbf{H}}] \neq 0$. As a result, the sudden cycles are characterized by the Von Neumann entropy $\mathcal{S}_{V N}=-\operatorname{tr}\{\hat{\boldsymbol{\rho}} \ln \hat{\boldsymbol{\rho}}\}$ being different from the energy entropy $\mathcal{S}_{E}=-\sum p_{n} \ln p_{n}$ where $p_{i}$ the expectation value of the $n$th energy level. $\mathcal{S}_{E} \geq \mathcal{S}_{V N}$ with equality only when $[\hat{\boldsymbol{\rho}}, \hat{\mathbf{H}}]=0$. These cycles are different from the time optimal bang bang type cycles which have significant off-diagonal elements in the energy representation on the adiabatic segments but are diagonal on the contact points [19, 24, 29, 30].

The large off diagonal elements of the density operator $\hat{\boldsymbol{\rho}}$ in the sudden cycles accompany their global behavior. The elements do not vanish on the connecting points between segments. As a result additional global links are generated. The interrelation of the observable values generate off diagonal elements of $\hat{\boldsymbol{\rho}}$. Increasing further the cycle time, the amplitude of the off-diagonal parts is suppressed. The coherence, defined in Sec. II characterizes the suddenness, showing that it decreases with increasing cycle time.

Another important property of the sudden cycles is that their performance is not necessarily optimal. Starting from an arbitrary initial state the relaxation toward a limit cycle of sudden cycle might take several thousands of iterations, as opposed to the regular cycles, where generally several iterations are sufficient. When the sudden cycle is closed, and leads to refrigeration, there is a close neighborhood of parameters which lead to similar cycles. These neighborhoods of analytic behavior are very small. As a result there are islands of 
parameters which lead to sudden refrigeration cycles which are disconnected from other islands.

To gain insight on the sudden cycles, analytical approximate expressions for the cooling power of the cycle, its coefficient of performance (COP), the coherence as a function of time, and the entropy generation of a cycle are obtained It is shown, both numerically and by analytic approximations, that the cooling power of the sudden cycles achieve a maximum value as a function of the inverse temperature multiplied by the coefficient of the inner coupling, as opposed to regular cycles which depend exponentially on the inverse temperature.

\section{THE CYCLE OF OPERATION, THE QUANTUM HEAT PUMP}

A heat pump extracts heat from a cold reservoir, and transfers it to the hot reservoir. The operation of the heat pump is determined by the properties of the working medium and the coupling to the cold and hot baths. The cycle of operation is defined by the external controls which include the variation in time of the field with the periodic property $\omega(t)=\omega(t+\tau)$ where $\tau$ is the total cycle time synchronized with the contact times on the different segments of the cycle. The cycle studied is composed of the following four segments, see Fig. 2:

1. Segment $A \rightarrow B$ (termed isomagnetic or isochore), the field is maintained constant $\omega=\omega_{c}$, the working medium is in contact with the cold bath of temperature $T_{c}$ with heat conductance $\Gamma_{c}$, and dephasing parameter $\gamma_{c}$ for a period of $\tau_{c}$, and with propagator $\mathcal{U}_{c}$.

2. Segment $B \rightarrow C$ (termed magnetization or compression adiabat), the field changes from $\omega_{c}$ to $\omega_{h}$ in a time period of $\tau_{c h}$ with propagator $\mathcal{U}_{c h}$.

3. Segment $C \rightarrow D$ isomagnetic, or isochore, the field is maintained constant $\omega=\omega_{h}$, the working medium is in contact with the hot bath of temperature $T_{h}$ with heat conductance $\Gamma_{h}$, and dephasing parameter $\gamma_{h}$ for a period of $\tau_{h}$ and with propagator $\mathcal{U}_{h}$.

4. Segment $D \rightarrow A$ demagnetization or expansion adiabat, the field changes from $\omega_{h}$ to $\omega_{c}$ in a time period of $\tau_{h c}$, with propagator $\mathcal{U}_{h c}$. 
In the basic paper, [10], section III describes in detail the definitions of the segments isochore and adiabat. A somewhat different approach can be found in the paper of Quan et al, [18].

At the limit cycle, all the values of the expectation values exactly repeat themselves during each cycle time $\tau$. The propagator of the cycle will be termed $\mathcal{U}_{\text {global }}$, where $\mathcal{U}_{\text {global }}$ is constructed by the individual propagators (for example at point $A$ in Fig. 22), as:

$$
\mathcal{U}_{\text {global }}=\mathcal{U}_{h c} \mathcal{U}_{h} \mathcal{U}_{c h} \mathcal{U}_{c}
$$

The limit cycle is characterized by an invariant eigenvector of $\mathcal{U}_{\text {global }}$, with eigenvalue 1 (one).

The dynamics of the refrigerator's working medium follow our previous studies [10, 28, 29]. We construct the segment propagators $\mathcal{U}$ by solving the dynamics for quantum thermodynamical observables. This dynamics is generated by completely positive maps within the formulation of quantum open systems [31, 32]. It is generated by the Liouville superoperator, $\mathcal{L}$ in the Heisenberg picture,

$$
\frac{d \hat{\mathbf{A}}}{d t}=i[\hat{\mathbf{H}}, \hat{\mathbf{A}}]+\mathcal{L}_{D}(\hat{\mathbf{A}})+\frac{\partial \hat{\mathbf{A}}}{\partial t}
$$

where $\mathcal{L}_{D}$ is a generator of a completely positive dissipative Liouville superoperator, which includes the temperatures $T_{c / h}$ of the reservoirs. The state of the system $\hat{\rho}$ is then reconstructed from a finite set of observables.

The Hamiltonian of the working fluid has the structure:

$$
\hat{\mathbf{H}}=\hat{\mathbf{H}}_{i n t}+\hat{\mathbf{H}}_{e x t}(t)
$$

where $\left[\hat{\mathbf{H}}_{i n t}, \hat{\mathbf{H}}_{e x t}(t)\right] \neq 0$. We chose a cycle that when the working fluid is in thermal contact with the reservoirs the Hamiltonian is stationary.

Motivated by a refrigerator operating with magnetic salt [3 6], a simple model was constructed with a working fluid composed of pairs of coupled spins. The uncontrolled, internal Hamiltonian becomes $(\hbar=1): \hat{\mathbf{H}}_{i n t}=\frac{1}{2} J\left(\hat{\boldsymbol{\sigma}}_{x}^{1} \otimes \hat{\boldsymbol{\sigma}}_{x}^{2}-\hat{\boldsymbol{\sigma}}_{y}^{1} \otimes \hat{\boldsymbol{\sigma}}_{y}^{2}\right) \equiv J \hat{\mathbf{B}}_{2} \quad$ where $\hat{\boldsymbol{\sigma}}$ represents the spin-Pauli operators, and $J$ scales the strength of the inter-particle interaction, which is assumed to be constant, for a given pump. When $J=0$, the model represents a working medium with noninteracting atoms [8]. The external Hamiltonian is chosen to be: $\hat{\mathbf{H}}_{e x t}=\frac{1}{2} \omega(t)\left(\hat{\boldsymbol{\sigma}}_{z}^{1} \otimes \hat{\mathbf{I}}^{\mathbf{2}}+\hat{\mathbf{I}}^{\mathbf{1}} \otimes \boldsymbol{\sigma}_{z}^{2}\right) \equiv \omega(t) \hat{\mathbf{B}}_{1} \quad$ where $\omega(t)$ represents the external control field. The total Hamiltonian becomes: $\hat{\mathbf{H}}=\omega(t) \hat{\mathbf{B}}_{\mathbf{1}}+\mathrm{J} \hat{\mathbf{B}}_{\mathbf{2}}$ 
The eigenvalues of $\hat{\mathbf{H}}$ are $\epsilon_{1}=-\Omega(t), \epsilon_{2 / 3}=0, \epsilon_{4}=\Omega(t)$ where $\Omega(t)=\sqrt{\omega(t)^{2}+J^{2}}$, which is the temporary energy scale, which is dependent on both the internal and external parts of the Hamiltonian. At various times $\hat{\mathbf{H}}(t)$ does not commute with itself since: $\left[\hat{\mathbf{B}}_{1}, \hat{\mathbf{B}}_{2}\right] \equiv 2 i \hat{\mathbf{B}}_{3} \neq 0, \quad \hat{\mathbf{B}}_{3}=\frac{1}{2}\left(\hat{\boldsymbol{\sigma}}_{y}^{1} \otimes \hat{\boldsymbol{\sigma}}_{x}^{2}+\hat{\boldsymbol{\sigma}}_{x}^{1} \otimes \hat{\boldsymbol{\sigma}}_{y}^{2}\right)$

The explicit equation for the dissipative part $\mathcal{L}_{D}(\hat{\mathbf{A}})[31]$ is

$$
\mathcal{L}_{D}(\hat{\mathbf{A}})=\sum_{\mathbf{j}}\left(\hat{\mathbf{F}}_{\mathrm{j}} \hat{\mathbf{A}} \hat{\mathbf{F}}_{\mathrm{j}}^{\dagger}-\frac{\mathbf{1}}{\mathbf{2}}\left(\hat{\mathbf{F}}_{\mathrm{j}} \hat{\mathbf{F}}_{\mathrm{j}}^{\dagger} \hat{\mathbf{A}}+\hat{\mathbf{A}} \hat{\mathbf{F}}_{\mathrm{j}} \hat{\mathbf{F}}_{\mathrm{j}}^{\dagger}\right)\right),
$$

where the $\left(\hat{\mathbf{F}}_{\mathrm{j}}\right)$ were identified with the raising and lowering operators, from energy level $n$ to $n-1$ and vice versa. These operators were constructed by first diagonalizing the Hamiltonian, then defining the $\left(\hat{\mathbf{F}}_{\mathrm{j}}\right)$ operators in the energy representation. The lowering transition rates $\kappa_{c / h}^{\downarrow}$ were chosen to be equal for all the four transitions, while the raising transition rates $\kappa_{c / h}^{\uparrow}$ were obtained by forcing detailed balance relation on the hot and cold isomagnetic segments $\kappa_{c / h}^{\uparrow} / \kappa_{c / h}^{\downarrow}=\exp \left(-\Omega_{c / h} / T_{c / h}\right)$. The heat transfer rate to the baths will be $\Gamma_{c / h}=\kappa_{c / h}^{\downarrow}+\kappa_{c / h}^{\uparrow},\left(k_{B}=1\right)$ [10], see also the Appendix.

In addition a dissipative generator of elastic encounters is added described as

$$
\mathcal{L}_{D^{e}}(\hat{\mathbf{A}})=-\gamma[\hat{\mathbf{H}},[\hat{\mathbf{H}}, \hat{\mathbf{A}}]]
$$

where $\gamma$ is the dephasing constant.

The state of the system $\hat{\boldsymbol{\rho}}$ can be expanded by a complete set of orthogonal operators on the Hilbert space of the system. The expansion coefficients are proportional to the expectation values of these operators. This set can form a complete vector space to represent the propagators $\mathcal{U}_{i}$ on different segments.

A thermodynamically inspired set of observables is a minimum set of operators which completely defines the state of the working medium when it reaches the limit cycle. For thermal equilibrium the energy and identity operators are sufficient. For the limit cycle this set has to be expanded. The set is initiated from the energy $\hat{\mathbf{H}}$ and new operators are added which are dynamically coupled to the energy. This set is formed from a linear combinations of the stationary closed set $\{\hat{\mathbf{B}}\}$ of operators:

$$
\hat{\mathbf{H}}=\omega(t) \hat{\mathbf{B}}_{1}+J \hat{\mathbf{B}}_{2} \hat{\mathbf{L}}=-J \hat{\mathbf{B}}_{1}+\omega(t) \hat{\mathbf{B}}_{2} \hat{\mathbf{C}}=\Omega(t) \hat{\mathbf{B}}_{3}
$$

The three operators defined in Eq. (6) form a closed Lie algebra, for they are linear combinations of the original operators $\hat{\mathbf{B}}_{\mathbf{i}}$, which also form a closed Lie algebra. 
To uniquely define the diagonal part of the state $\hat{\boldsymbol{\rho}}$ in the energy representation, the original set has to be supplemented with two additional operators: $\hat{\mathbf{V}}=\Omega \hat{\mathbf{B}}_{4}=\frac{1}{2} \Omega\left(\hat{\mathbf{I}}^{1} \otimes\right.$ $\hat{\boldsymbol{\sigma}}_{z}^{2}-\hat{\mathbf{I}}^{2} \otimes \hat{\boldsymbol{\sigma}}_{z}^{1}$ ) and $\hat{\mathbf{D}}=\Omega \hat{\mathbf{B}}_{5}=\Omega \hat{\boldsymbol{\sigma}}_{z}^{1} \otimes \hat{\boldsymbol{\sigma}}_{z}^{2}$. With this operator base the state $\hat{\boldsymbol{\rho}}$ can be expanded as:

$$
\hat{\boldsymbol{\rho}}=\frac{1}{4} \hat{\mathbf{I}}+\frac{1}{\Omega}(\langle\hat{\mathbf{H}}\rangle \hat{\mathbf{H}}+\langle\hat{\mathbf{L}}\rangle \hat{\mathbf{L}}+\langle\hat{\mathbf{C}}\rangle \hat{\mathbf{C}}+\langle\hat{\mathbf{V}}\rangle \hat{\mathbf{V}}+\langle\hat{\mathbf{D}}\rangle \hat{\mathbf{D}})
$$

$\hat{\mathbf{V}}$ and $\hat{\mathbf{D}}$ commute with $\hat{\mathbf{H}}$. The equilibrium value of $\langle\hat{\mathbf{V}}\rangle$ is zero, and once it reaches equilibrium it does not change during the cycle dynamics. This means that on the limit cycle the state $\hat{\boldsymbol{\rho}}$ can be reconstructed by four expectation values: $E=\langle\hat{\mathbf{H}}\rangle, L=\langle\hat{\mathbf{L}}\rangle$, $C=\langle\hat{\mathbf{C}}\rangle$ and $D=\langle\hat{\mathbf{D}}\rangle$. In the energy representation the state $\hat{\boldsymbol{\rho}}$ becomes:

$$
\hat{\boldsymbol{\rho}}_{e}=\frac{1}{4}\left(\begin{array}{cccc}
1+\frac{1}{\Omega}(D-2 E) & 0 & 0 & \frac{2}{\Omega}(L+i C) \\
0 & 1-\frac{1}{\Omega} D & 0 & 0 \\
0 & 0 & 1-\frac{1}{\Omega} D & 0 \\
\frac{2}{\Omega}(L-i C) & 0 & 0 & 1+\frac{1}{\Omega}(D+2 E)
\end{array}\right)
$$

A measure of the off diagonal elements in the energy frame is the coherence [33]:

$$
\tilde{\mathcal{C}}=\operatorname{tr}\left\{\left(\hat{\boldsymbol{\rho}}_{e}-\hat{\boldsymbol{\rho}}_{e d}\right)^{2}\right\}
$$

where $\hat{\boldsymbol{\rho}}_{e d}$ is the diagonal stationary part of the density operator in the energy frame. ¿From Eq. (8), the coherence becomes $\tilde{\mathcal{C}}=\frac{L^{2}+C^{2}}{2 \Omega^{2}}$. Fig. 3 shows the transition from a sudden cycle to a regular one on the adiabatic segment. An increase in the time allocation on this segment decrease the coherence $\tilde{\mathcal{C}}$.

The vector space defining the propagators is constructed from the four operators $\hat{\mathbf{H}}, \hat{\mathbf{L}}, \hat{\mathbf{C}}, \hat{\mathbf{D}}$ and the identity $\hat{\mathbf{I}} \vec{X}=(\hat{\mathbf{H}}, \hat{\mathbf{L}}, \hat{\mathbf{C}}, \hat{\mathbf{D}}, \hat{\mathbf{I}})$. Using this set the propagator $\mathcal{U}_{i}(\tau)$ on the isochores (or equivalently on the isomagnets) becomes [10];

$$
\mathcal{U}_{i}=\left(\begin{array}{ccccc}
e^{\left(-\Gamma_{c / h} \tau\right)} & 0 & 0 & 0 & E_{e q}\left(1-e^{\left(-\Gamma_{c / h} \tau\right)}\right) \\
0 & K \cos (\Omega \tau) & -K \sin (\Omega \tau) & 0 & 0 \\
0 & K \sin (\Omega \tau) & K \cos (\Omega \tau) & 0 & 0 \\
\frac{1}{\Omega}\left(E_{e q}\left(e^{-\Gamma_{c / h} \tau}-e^{-2 \Gamma_{c / h} \tau}\right)\right) & 0 & 0 & e^{-2 \Gamma_{c / h} \tau} & -\frac{E_{e q}^{2}}{\Omega}\left(e^{-\Gamma_{c / h} \tau}-1\right) \\
0 & 0 & 0 & 0 & 1
\end{array}\right)
$$

where $K=e^{\left(-\left[\Gamma_{c / h}+\gamma_{c / h} \Omega^{2}\right] \tau\right)}, \quad \Gamma_{c / h}, \gamma_{c / h}, \Omega, \tau$ are defined above.

The periodic functions in Eq. (10) mean that the isomagnetic segments are quantized. Whenever $\Omega \tau=2 \pi$, the two coupled equations of $L, C$ complete a period. The other two 

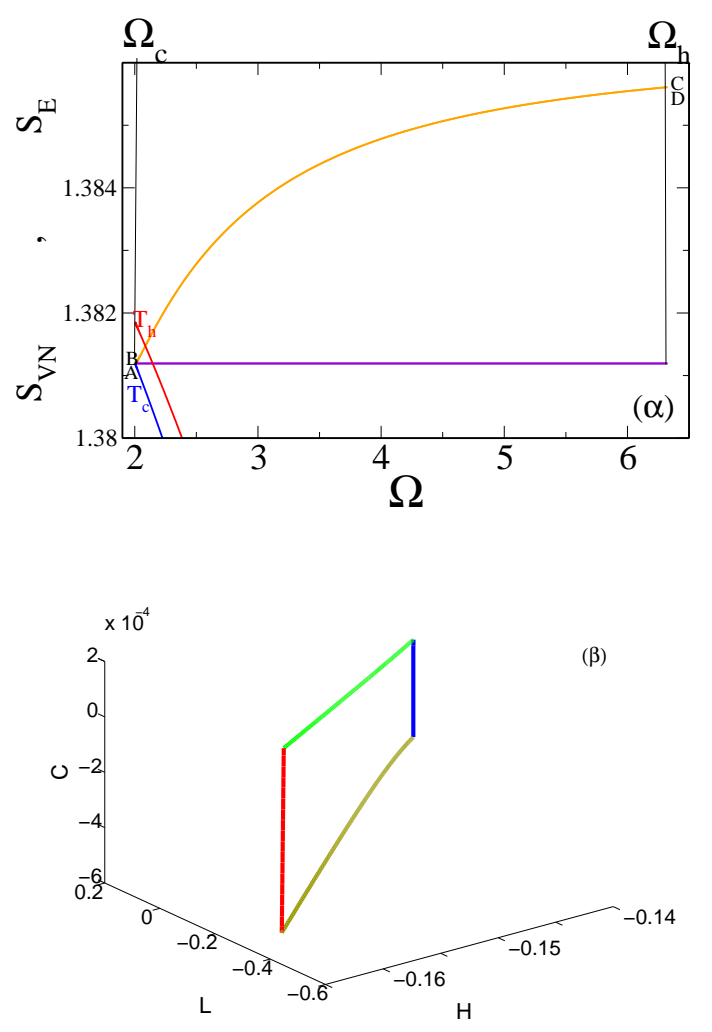

FIG. 1: (Color online)Top $(\alpha)$ : An example of an extreme sudden cycle with analytical scheduling, in the $\left(\Omega, S_{E}\right)$ plane (curved shape ) and $\left(\Omega, S_{V N}\right)$ plane (shrinked rectangle shape), together with the isotherms corresponding to the cold/hot baths temperatures, $T_{c} / T_{h}$. The cooling power $Q_{c} / \tau=1.2 \cdot 10^{-6}$. The cyle parameters are: $T_{c}=14, T_{h}=15, J=2 ., \omega_{c}=0.1, \omega_{h}=6$. . The time allocations: $\tau_{c}, \tau_{c h}, \tau_{h}, \tau_{h c}=0.9,0.00035,0.00025,0.00035, \kappa_{h}^{\downarrow}=0.36, \kappa_{c}^{\downarrow}=0.328, \operatorname{Bottom}(\beta)$ : the corresponding cycle trajectory in the $H, L, C$ space.

expectation values $E$ and $D$ are decoupled from $L, C$ the expectation values of the operators $\hat{\mathbf{L}}$ and $\hat{\mathbf{C}}$. Quantization exists also on the adiabats. Closed form solutions for the adiabats leading to quantized motion are obtaind for a constant adiabatic parameter $\mu=\frac{J \dot{\omega}}{\Omega^{3}}[29]$.

Sudden cycles can be classified according to the time spent on the different branches. Fig. 1 displays the most extreme case. It seems that the two magnetization and demagnetization adiabats almost coincide in the entropy frequency plane. Also one recognizes that the cold isotherm almost touches the only point where the $S_{E}$ and $S_{V N}$ meet, showing that further cooling is impossible. The Von Neumann entropy $S_{V N}=-\operatorname{tr}\{\hat{\boldsymbol{\rho}} \ln \hat{\boldsymbol{\rho}}\}$ is constant on the 

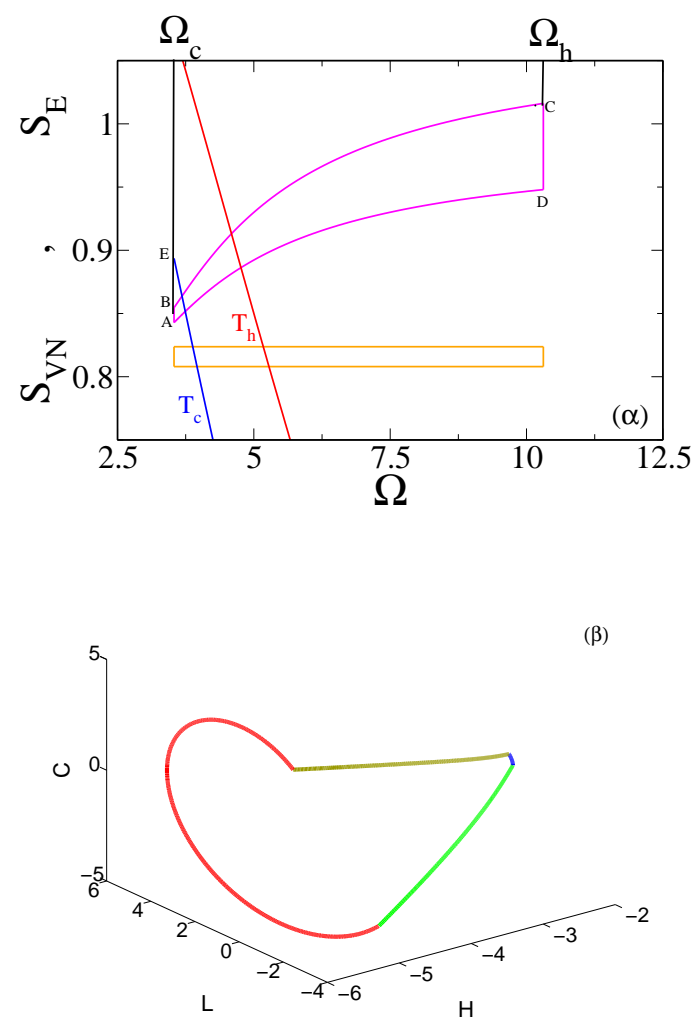

FIG. 2: (Color online)A typical sudden cycle of the refrigerator with additional time allocation on the segments. $\operatorname{Top}(\alpha)$ : in the $\left(\Omega, \mathcal{S}_{E}\right)$, and $\left(\Omega, \mathcal{S}_{V N}\right)$ plane (rectangle), together with the isotherms corresponding to the cold/hot baths temperatures, $T_{c} / T_{h}$. Note $\mathcal{S}_{E}>\mathcal{S}_{V N}$. The cycle parameters are: $T_{c}=2.175, T_{h}=2.9, J=2.5, \omega_{c}=2.5, \omega_{h}=10$. The time allocations are: $\tau_{c}, \tau_{c h}, \tau_{h}, \tau_{h c}=$ $0.2,0.21,0.44,0.21, \kappa_{h, c}=0.36,0.328$. $\operatorname{Bottom}(\beta)$ : the corresponding cycle trajectory in the $H, L, C$ space. The longer time allocation on the hot isomagnetic segment and the larger frequency $\Omega_{h}$ allows to complete approximately $3 / 4$ of a period.

adiabats since the dynamics on these segments is unitary. The bottom of Fig. 1, shows the trajectories in the $H, L, C$ space indicating that the dependence of the different segments forces the trajectory to reside on a plane.

Fig. 2 shows a second type of sudden cycle with addional time allocation on the isomagnetic segments. The bottom of Fig. 2 shows the trajectory of the cycle in the $H, L, C$ space. One notices the global property of the cycle, by realizing that the isomagnetic segments complement each other (see also the third sudden cycle, Fig. 4). The rotation of $L$ and $C$ 


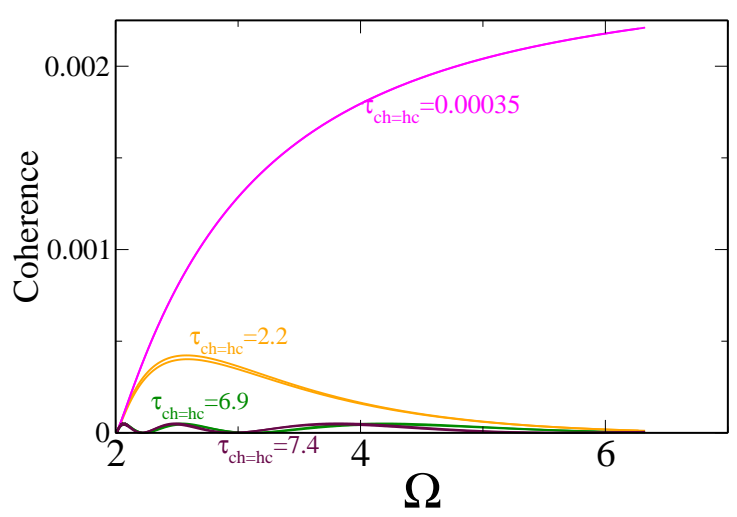

FIG. 3: (Color online)The coherence $\tilde{\mathcal{C}}$, Eq. (9) as a function of $\Omega$ for cycles with different time allocations on the adiabats. The cycle parameters correspond to the data of Fig. 1 except the different $\tau_{h c}=\tau_{c h}$. Notice the decrease in coherence when the time allocation on the adiabats increases.

complete one period split between the hot and cold isomagnetic segments. In addition, Fig. 2 shows that further cooling is possible, because the end point $B$ of the cold isomagnetic segment is below the point $E$ where the vertical $B E$ intersects with the cold isotherm. Point $E$ is the upper-bound on $S_{E}$ for a cooling cycle. The maximum heat per cycle that still can be extracted from the cold reservoir is $T_{c}\left(\mathcal{S}_{E}(E)-\mathcal{S}_{E}(B)\right)$.

One should also notice that in all the sudden cycles, the significant difference between $S_{E}^{\max }-S_{E}^{\min }$ and $S_{V N}^{\max }-S_{V N}^{\min }$, or generally the difference between $S_{E}$ and its corresponding lower bound $S_{V N}$, all of which indicate the large off diagonal elements of the density matrix in the energy representation. The reason is that while $H, L, C$ are created on the adiabats, the very short time on the hot isochores is not sufficient to equilibrate the energy of the working fluid. Generally, the time on the segments is so short, that less than one period is achieved.

\section{ANALYTICAL APPROXIMATIONS FOR THE COOLING POWER.}

The cooling power is the object of this study. The amount of heat extracted from the cold bath is defined by the difference in energy of the working medium between point $B$ and point $A$ Cf. Fig. 2. The cooling power, $\mathcal{P}_{c}=Q_{c} / \tau$, is the heat flow from the cold reservoir 

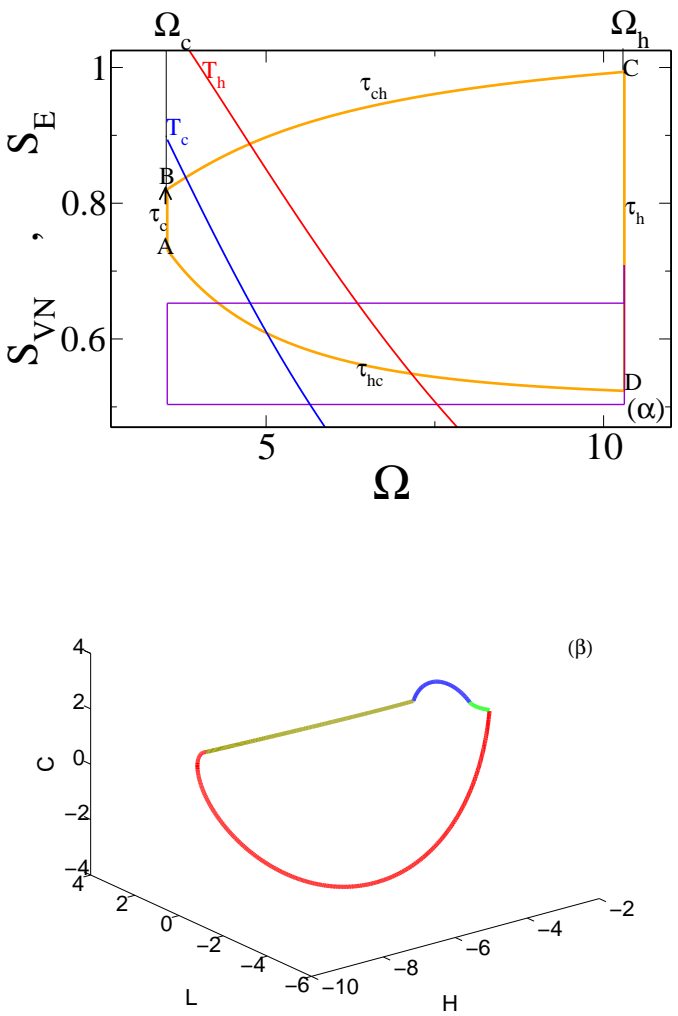

FIG. 4: (Color online) A sudden cycle with additional time allocated on the isomagnetic segments. The cycle parameters are: $\mathrm{J}=2.5, T_{h}=2.9, T_{c}=2.175, \omega_{h}=10, \omega_{c}=2.5 k_{h} \downarrow=3.35, k_{c} \downarrow=0.328$ $\tau_{h}=0.442, \tau_{h c}=0.00744, \tau_{c}=0.527, \tau_{c h}=0.00824 . \operatorname{Top}(\alpha):$ In the $\left(\Omega, \mathcal{S}_{E}\right),\left(\Omega, \mathcal{S}_{V N}\right)$ planes. $\operatorname{Bottom}(\beta)$ : The corresponding trajectory in the $H, L, C$ space. Linear scheduling of $\omega(t)$.

into the system divided by $\tau$.

$$
\mathcal{P}_{c}=\mathcal{Q}_{c} / \tau=\left(E_{B}-E_{A}\right) / \tau \text {. }
$$

The values of $E_{A}$ and $E_{B}$ are calculated from the limit cycle invariant vector $\vec{X}$ of the cycle propagator $\mathcal{U}_{c y c}$ at these points.

The commutator of the cycles' propagators:

$$
\left[\mathcal{U}_{A B}, \mathcal{U}_{c}\right]
$$

where $\mathcal{U}_{A B}=\mathcal{U}_{h c} \mathcal{U}_{h} \mathcal{U}_{c h}$ supplies an indication of the cooling power. When $\left[\mathcal{U}_{A B}, \mathcal{U}_{c}\right]=0$, $\mathcal{Q}_{c}=0$. This relation is used to check approximations of the propagators $\mathcal{U}$. The sudden 
limit on the different segments suggest a short time approximation. We use the commutator Eq. (12) to check the minimum order of the approximation.

\section{A. Approximations for the adiabats}

The exact solution for the propagator on the adiabats, $\mathcal{U}_{a d}$ for a constant adiabatic parameter $\mu$ has been derived in Ref. [29], Eq. (18):

$$
\mathcal{U}_{a d}=\frac{\Omega_{c}}{\Omega_{h}}\left(\begin{array}{ccccc}
\frac{1+\mu^{2} c}{q^{2}} & -\frac{\mu s}{q} & \frac{\mu(1-c)}{q^{2}} & 0 & 0 \\
\frac{\mu s}{q} & c & -\frac{s}{q} & 0 & 0 \\
\frac{\mu(1-c)}{q^{2}} & \frac{s}{q} & \frac{\mu^{2}+c}{q^{2}} & 0 & 0 \\
0 & 0 & 0 & 1 & 0 \\
0 & 0 & 0 & 0 & \frac{\Omega_{h}}{\Omega_{c}}
\end{array}\right),
$$

where $q=\sqrt{1+\mu^{2}}, s=\sin (q \Theta)$ and $c=\cos (q \Theta)$. The angle $\Theta$ for the hot-to-cold adiabat is defined as:

$$
\Theta_{h c}=\tau_{a d i}\left(1 / K_{h c}\right)\left(\arcsin \left(\omega_{c} / \Omega_{c}\right)-\arcsin \left(\omega_{h} / \Omega_{h}\right)\right) \equiv \frac{\tau_{a d i}}{K_{h c}}\left(\Phi_{h c}\right)
$$

A similar expression is obtained for cold-to-hot angle $\Theta_{c h}$, where: $K_{h c}=\frac{1}{J}\left(\omega_{c} / \Omega_{c}-\omega_{h} / \Omega_{h}\right)=$ $-K_{c h}=-\frac{1}{J}\left(\omega_{h} / \Omega_{h}-\omega_{c} / \Omega_{c}\right)$. The propagator Eq. (13) is proportional to the compression ratio: $\mathcal{C}=\frac{\Omega_{c}}{\Omega_{h}}$ except for the last term which corresponds to the identity.

Expanding the terms composing $\mathcal{U}_{h c}$ for short time $\left(\tau_{c h}=\tau_{h c} \equiv \tau_{a d i} \ll 1\right)$, one obtains $|\mu|=q$. This result follows from the definitions of $\mu$ and $q$ : $\mu_{h c}=-\mu_{c h}=\frac{K_{h c}}{\tau_{a d i}}=-\frac{K_{c h}}{\tau_{a d i}}$, where the relation between $\mu$ and $\tau_{a d i}$ is obtained from [29]. Since $\tau_{a d i} \ll 1$, from the definition of $\mu$ one obtains that $|\mu| \gg 1$. Further: $q=\sqrt{1+\mu^{2}}$. Therefore $|\mu|=q$. Under these conditions $\mathcal{U}_{h c}$, Eq. (13), is approximated as:

$$
\mathcal{U}_{h c}^{(a p p r)} \approx \frac{\Omega_{c}}{\Omega_{h}}\left(\begin{array}{ccccc}
c & -s & 0 & 0 & 0 \\
s & c & 0 & 0 & 0 \\
0 & 0 & 1 & 0 & 0 \\
0 & 0 & 0 & 1 & 0 \\
0 & 0 & 0 & 0 & \frac{\Omega_{h}}{\Omega_{c}}
\end{array}\right)
$$

The explicit expression for the argument $q \Theta$ becomes: $q \Theta=|\mu| \Theta=|K| / \tau_{a d i} \Theta=$ $|K| / \tau_{a d i} \frac{\tau_{a d i}}{|K|}\left(\Phi_{h c}\right)=\left(\Phi_{h c}\right)$. A similar expression is obtained for $\mathcal{U}_{c h}^{(a)}$. Notice that in Eq. (15), only the vector components $\hat{\mathbf{H}}$ and $\hat{\mathbf{L}}$ exchange their values. 
1. Further classification of sudden approximations on the adiabats .

A further classification of the propagators on the adiabats is defined according to the values of the operating parameters $\omega_{c}, \omega_{h}$ and $J$ is based on additional simplification of either Eq. (13) or Eq. (15).

1. When $\omega_{c} \ll J, \omega_{h} \gg J$ and also $\tau_{a d i} \ll \frac{2 \pi}{\Omega(t)}$ in the range of $\mathrm{t}$, then the propagator $\mathcal{U}_{h c}^{(a p p r)}$ will simplify to the form:

$$
\mathcal{U}_{h c}^{(1)}=\approx \frac{\Omega_{c}}{\Omega_{h}}\left(\begin{array}{ccccc}
0 & 1 & 0 & 0 & 0 \\
-1 & 0 & 0 & 0 & 0 \\
0 & 0 & 1 & 0 & 0 \\
0 & 0 & 0 & 1 & 0 \\
0 & 0 & 0 & 0 & \frac{\Omega_{h}}{\Omega_{c}}
\end{array}\right)
$$

2. When $\omega_{c} \sim J, \omega_{h} \gg J$ and also $\tau_{a d i} \ll \frac{2 \pi}{\Omega(t)}$. In this case $\mathcal{U}_{h c}^{(a p p r)}$ will have the form :

$$
\mathcal{U}_{h c}^{(2)} \approx \frac{\Omega_{c}}{\Omega_{h}}\left(\begin{array}{ccccc}
\frac{1}{\sqrt{2}} & \frac{1}{\sqrt{2}} & 0 & 0 & 0 \\
-\frac{1}{\sqrt{2}} & \frac{1}{\sqrt{2}} & 0 & 0 & 0 \\
0 & 0 & 1 & 0 & 0 \\
0 & 0 & 0 & 1 & 0 \\
0 & 0 & 0 & 0 & \frac{\Omega_{h}}{\Omega_{c}}
\end{array}\right)
$$

3. $\operatorname{case}(\mathrm{a})$

When $\omega_{c}, \omega_{h} \gg J$ and also $\tau_{a d i} \ll \frac{2 \pi}{\Omega(t)}$. then $\mathcal{U}_{h c}^{(a p p r)}$ becomes:

$$
\mathcal{U}_{h c}^{(3 a)} \approx \frac{\Omega_{c}}{\Omega_{h}}\left(\begin{array}{ccccc}
1 & 0 & 0 & 0 & 0 \\
0 & 1 & 0 & 0 & 0 \\
0 & 0 & 1 & 0 & 0 \\
0 & 0 & 0 & 1 & 0 \\
0 & 0 & 0 & 0 & \frac{\Omega_{h}}{\Omega_{c}}
\end{array}\right)
$$

Case(b)

When $\omega_{c}, \omega_{h} \gg J$ and also $\tau_{a d i} \approx 1$.

¿From the condition $\omega_{c}, \omega_{h} \gg J$ the ratio $\frac{\omega}{\Omega}$ becomes:

$$
\frac{\omega}{\Omega}=1-(1 / 2)\left(\frac{J}{\omega}\right)^{2} .
$$


¿From the definition of $\mu$ in Eq (13), $K_{\text {adi }}$ after Eq. (14) and condition (b), follows $K_{\text {adi }}<1$, therefore $\mu<1$. In addition from the definition of $q$ follows $q=1$. Therefore Eq. (13) simplifies by neglecting $\mu^{2}$ to the following propagator:

$$
\mathcal{U}_{h c}^{(3 b)}=\frac{\Omega_{c}}{\Omega_{h}}\left(\begin{array}{ccccc}
1 & -\mu s & \mu(1-c) & 0 & 0 \\
\mu s & c & -s & 0 & 0 \\
\mu(1-c) & s & c & 0 & 0 \\
0 & 0 & 0 & 1 & 0 \\
0 & 0 & 0 & 0 & \frac{\Omega_{h}}{\Omega_{c}}
\end{array}\right)
$$

In ref. [29], when expanding $H, L, C$ in Eq. (13) to first order in $\mu$, a propagator similar to Eq. (20) was obtained.

¿From $q=1$, Eqs. (19) and (14), the argument $q \Theta$ of the trigonometric functions becomes

$$
q \Theta_{h c}=1 \tau_{a d i} \frac{\left(J^{2} / 2\right)\left(1 / \omega_{h}^{2}-1 / \omega_{c}^{2}\right)}{(J / 2)\left(1 / \omega_{h}^{2}-1 / \omega_{c}^{2}\right)}=\tau_{a d i} J
$$

In this case the argument $q \Theta=\Theta$ of the trigonometric functions is large therefore it cannot be approximated to first (or second) order.

\section{B. Approximations for the isomagnetic segments .}

For the isomagnetic segments the propagators $\mathcal{U}_{c}$ and $\mathcal{U}_{h}$ are approximated as follows.

1. First we assume that the time allocation on both isomagnetic segments is short enough, so that in Eq. (10), $\sin (\Omega \tau)=0$, and $\cos (\Omega \tau)=1$. In addition, $\gamma=0$. Then Eq. (10) simplifies to:

$$
\mathcal{U}_{i}^{1} \approx\left(\begin{array}{ccccc}
e^{\left(-\Gamma_{c / h} \tau\right)} & 0 & 0 & 0 & E_{e q}\left(1-e^{\left(-\Gamma_{c / h} \tau\right)}\right) \\
0 & e^{\left(-\Gamma_{c / h} \tau\right)} & 0 & 0 & 0 \\
0 & 0 & e^{\left(-\Gamma_{c / h} \tau\right)} & 0 & 0 \\
\frac{1}{\Omega}\left(E_{e q}\left(e^{-\Gamma_{c / h} \tau}-e^{-2 \Gamma_{c / h} \tau}\right)\right) & 0 & 0 & e^{-2 \Gamma_{c / h} \tau} & \frac{E_{e q}^{2}}{\Omega}\left(e^{-\Gamma_{c / h} \tau}-1\right) \\
0 & 0 & 0 & 0 & 1
\end{array}\right)(22)
$$

2. On the isomagnetic segments we assume short time on the hot isomagnetic segment, leading to a first order approximation in time. The result is an approximate propagator 
for the hot isomagneticic segment:

$$
\mathcal{U}_{h o t}^{2} \approx\left(\begin{array}{ccccc}
1-\Gamma_{h} \tau_{h} & 0 & 0 & 0 & \left(E_{h}^{e q}\right)\left(\Gamma_{h} \tau_{h}\right) \\
0 & 1-\Gamma_{h} \tau_{h} & -\Omega_{h} \tau_{h} & 0 & 0 \\
0 & \Omega_{h} \tau_{h} & 1-\Gamma_{h} \tau_{h} & 0 & 0 \\
\left(\frac{1}{\Omega_{h}}\right)\left(E_{h}^{e q}\right)\left(\Gamma_{h} \tau_{h}\right) & 0 & 0 & 1-2 \Gamma_{h} \tau_{h} & \frac{E_{h}^{e q}}{\Omega_{h}}\left(\Gamma_{h} \tau_{h}\right) \\
0 & 0 & 0 & 0 & 1
\end{array}\right)
$$

A first order approximation also on the cold segment leads to the commutation of the segment propagators Eq. (12) to vanish $\left[\mathcal{U}_{A B}, \mathcal{U}_{c}\right]=0$. Therefore for the cold segment, the unapproximated Eq. (10) is employed.

Another possibility is that the hot and cold isomagnetic segments are swapped, and the time on the cold isomagnetic segment is short, while the hot isomagnetic is not approximated.

3. In this case both of the isomagnetic segments are approximated to the second order in time leading to:

$$
\mathcal{U}_{c / h}^{3} \approx
$$

$$
\left(\begin{array}{ccccc}
1-G+\frac{G^{2}}{2} & 0 & 0 & 0 & E^{e q}\left(G-\frac{G^{2}}{2}\right) \\
0 & 1-G+\frac{\left(\Gamma^{2}-\Omega^{2}\right) \tau^{2}}{2} & -\Omega \tau+\Gamma \Omega \tau^{2} & 0 & 0 \\
0 & \Omega \tau-\Gamma \Omega \tau^{2} & 1-G+\frac{\left(\Gamma^{2}-\Omega^{2}\right) \tau^{2}}{2} & 0 & 0 \\
\frac{E^{e q}}{\Omega}\left(G-\frac{3 G^{2}}{2}\right) & 0 & 0 & 1-2 G+2 G^{2} & \frac{E^{e q 2}}{\Omega}\left(G-\frac{G^{2}}{2}\right) \\
0 & 0 & 0 & 0 & 1
\end{array}\right)
$$

and $G=\Gamma \tau$.

\section{The approximate cooling power}

The heat removed from the cold bath at each period is calculated from the global propagator, Eq. (11). The approximations of the segment propagators are employed, Eqs. (16) to (24), to obtain a global propagator. The next step is to evaluate the invariant vector of the global propagator with eigenvalue one. Obtaining such a vector is an internal verification on the validity of the approximation. The energy $E$ component of the eigenvector at two points $B$ and $A$ in Fig. 2 lead to $\mathcal{Q}_{c}=E_{B}-E_{A}$. 
1. In the first combination the adiabats will be approximated by Eq. (16), while the hot isomagnetic segment will be approximated by Eq. (23), and the cold isomagnetic by Eq. (10). Denoting $e^{-\left(\Gamma_{c} \tau_{c}\right)}=\alpha$ and $\cos \left(\Omega_{c} \tau_{c}\right)=c c, \sin \left(\Omega_{c} \tau_{c}\right)=s s$, the heat removed from the cold bath in this approximation, $\mathcal{Q}_{c}^{\text {appr1 }}$ becomes:

$$
\mathcal{Q}_{c}^{a p p r 1} \approx-\frac{\alpha \tau_{h}^{2}\left(\Omega_{c} s s E_{h}^{e q} \Gamma_{h}-\Omega_{h}^{2} E_{c}^{e q} c c+E_{c}^{e q} \alpha \Omega_{h}^{2}\right)}{\left(\alpha^{2}-2 \alpha c c+1\right)}+\frac{\left(2 \alpha \tau_{h}^{2} \Gamma_{h}^{2} E_{c}^{e q}(\alpha-c c)\right)}{(\alpha-1)}
$$

The second term in Eq. (25) is typically two order of magnitude smaller than the first term, therefore we neglect it. The final approximation in this case becomes:

$$
\mathcal{Q}_{c}^{a p p r 1 b} \approx-\frac{\alpha \tau_{h}^{2}\left(\Omega_{c} s s E_{h}^{e q} \Gamma_{h}-\Omega_{h}^{2} E_{c}^{e q} c c+E_{c}^{e q} \alpha \Omega_{h}^{2}\right)}{\left(\alpha^{2}-2 \alpha c c+1\right)}
$$

The approximations of Eq. (25) and (26) correspond to the data of Fig. 11. The approximation was compared to the numerical calculations in the range of parameters corresponding to Fig. 1, in a neighborhood of $\tau_{c}$, and $T_{c}$. A good agreement was obtained up to a constant numerical factor.

The heat removed $\mathcal{Q}_{c}$ in Eq. (26) can change sign meaning that for certain values of parameters the refrigeration stops. Fig. 6 shows the alternating cooling as a function of $\tau_{c}$. The switching points of approximation Eq. (26) are conjectured at the points where the functions $\exp \left(\Gamma_{c} \tau_{c}\right)$ and $\cos \left(\Omega_{c} \tau_{c}\right)$ cut each other, (Bottom of Fig. 6). As can be seen these points form a good approximation.

Fig. 10 is a map of showing regions in parameter space where refrigeration takes place and regions where there is no refrigeration. In this map $\tau_{c}=\tau_{h}$, which is a border case of the approximation, because the approximation in Eq. (26) assumes $\tau_{c} / \tau_{h} \gg 1$.

2. In the second case the adiabats are approximated by Eq. (17). On both isomagnets a second order approximation is used, Eq. (24), leading to:

$$
\mathcal{Q}_{c}^{\text {appr } 2} \approx \frac{\left(E_{c}^{e q}-\frac{\Omega_{c} E_{h}^{e q}}{\Omega_{h}}\right)\left(\Gamma_{c} \tau_{c}-\frac{1}{2}\left(\Gamma_{c} \tau_{c}\right)^{2}\right)\left(\Gamma_{h} \tau_{h}-0.5\left(\Gamma_{h} \tau_{h}\right)^{2}\right)}{\left(\Gamma_{c} \tau_{c}+\Gamma_{h} \tau_{h}\right)-\frac{1}{2}\left(\Gamma_{c} \tau_{c}+\Gamma_{h} \tau_{h}\right)^{2}}
$$

Fig. 5 shows cycles corresponding to the conditions of Eq. (27), with the additional condition: $\omega_{h} \tau_{h}=$ constant $=6.252$. Comparison of the approximation of Eq. (27) to 

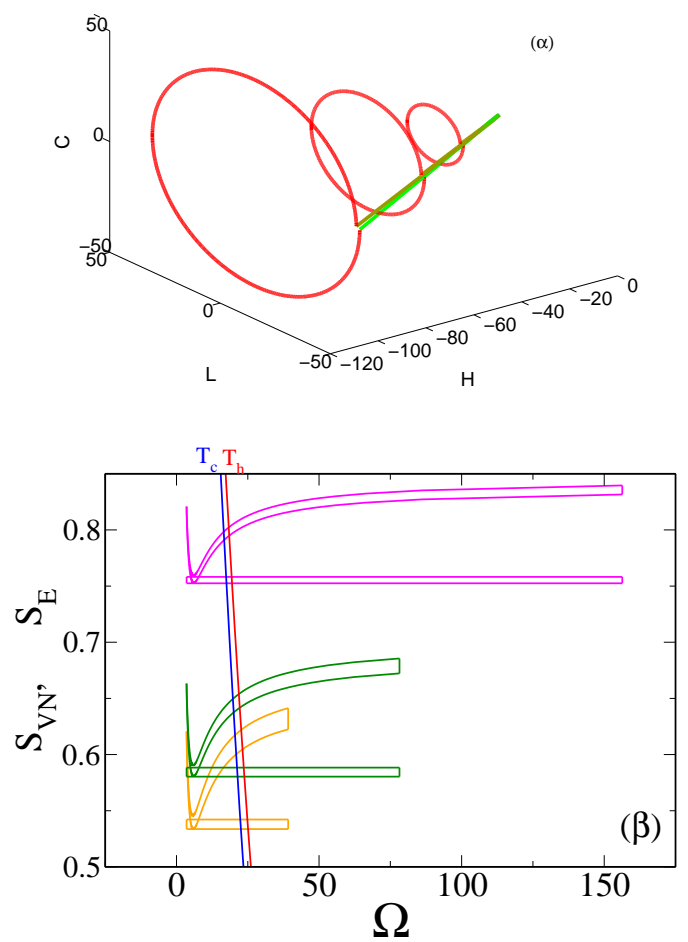

FIG. 5: (Color online) Top $(\alpha)$ : Cycle trajectories in the $H, L, C$ space which complete approximately one revolution on the hot isomagnetic segment. $\omega_{h} \tau_{h}=6.252$. The data for the three cycles are: $J=2.5, T_{h}=10, T_{c}=9 ., \omega_{c}=2.5 k_{h} \downarrow=0.36, k_{c} \downarrow=0.328 \tau_{h c}=\tau_{c h}=0.065625, \tau_{c}=$ 0.008375, ¿From large, to small cycles: $\omega_{h}=156.3,78.15,39.075$ and therefore $\tau_{h}=0.04,0.08,0.16$ $\operatorname{Bottom}(\beta)$ : The same cycles in the $\left(\Omega, \mathcal{S}_{E}\right),\left(\Omega, \mathcal{S}_{V N}\right)$, planes.

numerical values of $\mathcal{Q}_{c}$ show good agreement with deviations up to $\sim 20 \%$. Section $\nabla$ addresses a large subfamily of cycles corresponding to Eq. (27), with the additional condition of $\omega_{h} \tau_{h}=$ constant. see Fig. 11.

3. In the third approximation we will distinguish between two cases.

Case A;

First we combine Eq. (18) for the adiabats and Eq. (22) for the isomagnets. This case becomes equivalent (for $\left(\Gamma_{c} \tau_{c}+\Gamma_{h} \tau_{h}\right)<2$ ) to the frictionless cycles studied before [29]. 
This means that there is no coupling between the energy $E$ and the other variables $L$ and $C$. As a result for short times a bang-bang type solution is optimal [9]. Expanding the exponents to second order one gets the following expression:

$$
\mathcal{Q}_{c}^{a p p r 3 a} \approx E_{h} \frac{\left(\frac{E_{c}^{e q}}{E_{h}^{e q}}-\frac{\Omega_{c}}{\Omega_{h}}\right)\left(\Gamma_{c} \tau_{c} \Gamma_{h} \tau_{h}\right)}{\left(\Gamma_{c} \tau_{c}+\Gamma_{h} \tau_{h}\right)-\left(\Gamma_{c} \tau_{c}+\Gamma_{h} \tau_{h}\right)^{2}}
$$

It it can be shown using Eq. (A2) that $\mathcal{Q}_{c}^{\text {appr } 3} \geq 0$ for $\left(\Gamma_{c} \tau_{c}+\Gamma_{h} \tau_{h}\right)<2$.

Case B;

Combining Eq. (201) for the adiabats and Eq. (22) for the isomagnets leads to case B. Computing the eigenvalues of the corresponding global propagator, the variables $H_{A}$ and $H_{B}$ separates beautifully from the other operator expectation values. The result:

$$
\mathcal{Q}_{c}^{a p p r 3 b} \approx E_{h} \frac{\left(\frac{E_{c}^{e q}}{E_{h}^{e q}}-\frac{\Omega_{c}}{\Omega_{h}}\right)\left(\Gamma_{c} \tau_{c} \Gamma_{h} \tau_{h}\right)-2 \frac{E_{c}^{e q}}{E_{h}^{e q}}\left(1-A_{c}\right) A_{h} \mu^{2}(1-c)}{1-A_{h} A_{c}\left(1+2 \mu^{2}(1-c)\right)}
$$

where $c=\cos \left(J \tau_{\text {adi }}\right)$ and $A_{h / c}=\exp \left(-\Gamma_{h / c} \tau_{h / c}\right)$. Fig. 9 plots Eq. (29) as a function of $\tau_{a d i}$ with some internal scaling. The approximation demonstrates the sign changes of the heatflow. The cycles of Fig. 7 correspond to Eq. (29). In Section IIID we will further elaborate on the properties of those cycles and their approximations.

\section{The discontinuous character of the sudden cycle families}

An example of the discontinuous behavior is shown in Fig. 7 corresponding to Eq. (29). When the time allocation on the adiabats is increased the cycles changed from a concave shape to a convex shape. Two of these cycles are shown in Fig. 7. Additional reduction in cycle times leads to $\mathcal{Q}_{c}<0$, which means that the cycles cease to be refrigerators. Then by further reducing the allocated times, the cycles suddenly transformed into a concave shape. In addition $\mathcal{Q}_{c}>0$, refrigerator cycles again. Fig. 7 shows an example for the stated behavior of the sudden cycles; there are families of cycles with a small $\delta$ neighborhood, beyond which a discontinuity emerges. Changing parameters can lead to another small neighborhood. In Fig. 7 the times on the segments were changed proportionally, decreasing the overall cycle time, so that $\tau_{\text {cycle }} \rightarrow 0$. Fig. 8 presents the cycles of Fig. 7 in the $H, L, C$ space. 

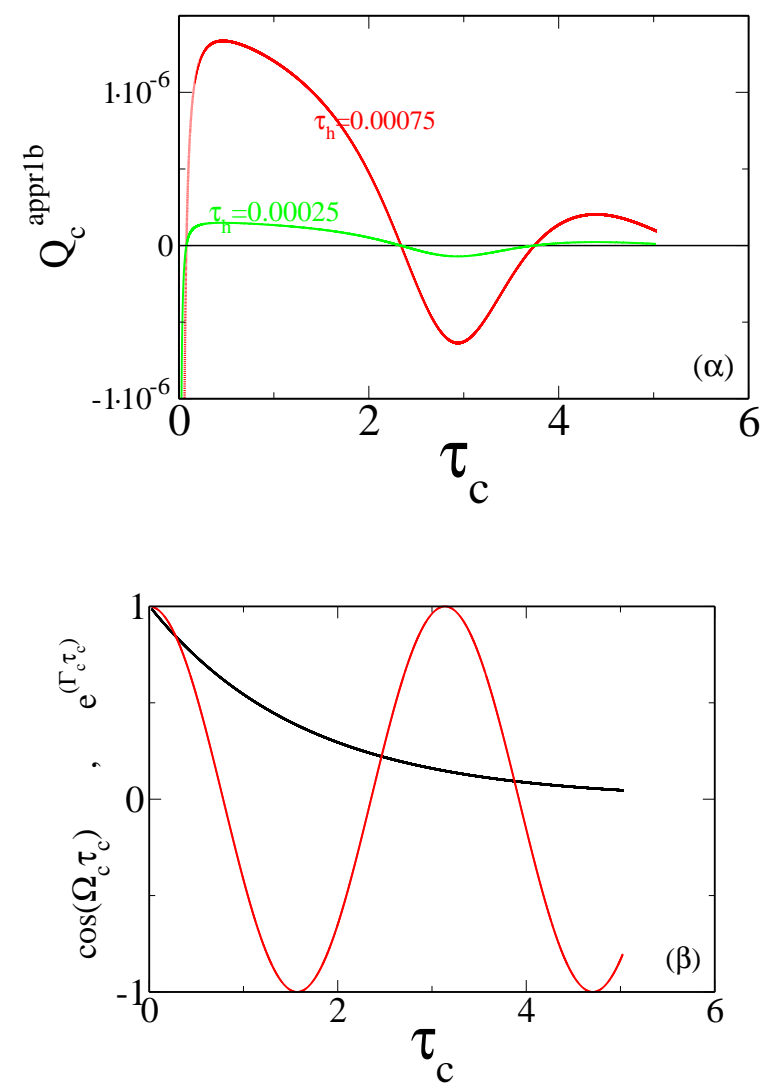

FIG. 6: (Color online) Top $(\alpha)$ : Eq. (26) is presented when changing the values of $\tau_{c}$, for two different $\tau_{h}$ values, as denoted on the figure: $\tau_{h}=0.00075$ red line , $\tau_{h}=0.00025$ green line. $\operatorname{Bottom}(\beta)$ : The functions $\exp \left(\gamma_{c} \tau_{c}\right)$ and $\cos \left(\Omega_{c} \tau_{c}\right)$ are shown as functions of $\tau_{c}$. Their crossing points compare well to the exact points(Top figure). The other parameters are $J=2, \tau_{c h}=\tau_{h c}=$ $0.00035, \omega_{c}=0.1, \omega_{h}=6, T_{h}=15, T_{c}=14$, as in Fig. 11.

\section{THE COEFFICIENT OF PERFORMANCE (COP) AND THE ENTROPY GENERATION $\left(\mathcal{S}^{u}\right)$}

The coefficient of performance (COP) is defined as the heat extracted divided by the work input:

$$
\mathrm{COP} \approx \frac{\mathcal{Q}_{\mathrm{c}}}{\mathcal{W}^{\mathrm{on}}}
$$



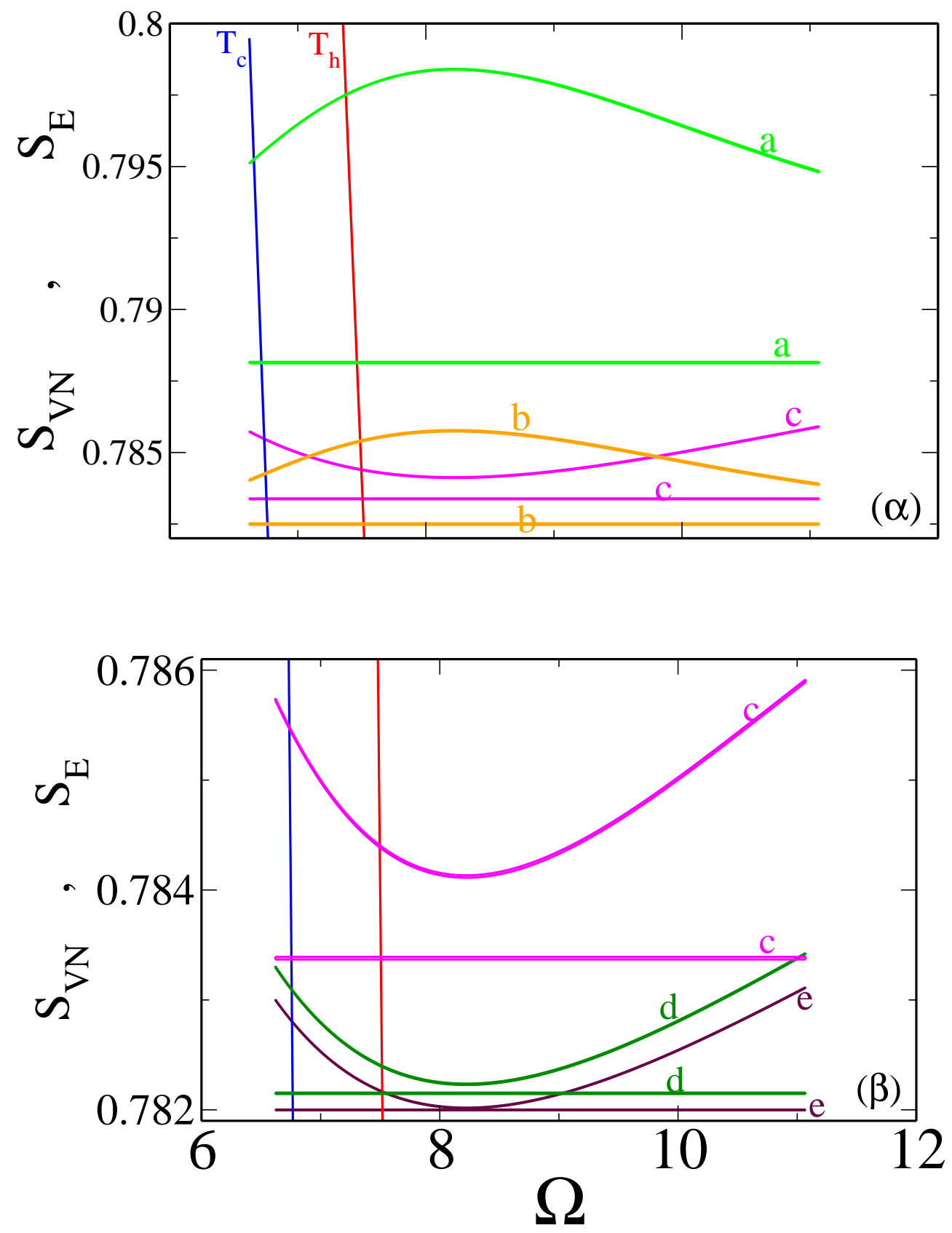

FIG. 7: (Color online) $\operatorname{Top}(\alpha): \mathcal{S}_{E}$ and $\mathcal{S}_{V N}$ as a function of $\Omega$ cycles for three different sets of times $, \tau_{h}, \tau_{c h}=\tau_{h c}, \tau_{c}=$ are: $\quad 0.000405,0.4194,0.029538$ green (a), 0.00045, 0.466, 0.03282 orange (b), 0.00036, 0.3728, 0.026256 non refrigeration cycles, 0.000225, 0.233, 0.01641 magenta (c) cycles. Bottom $(\beta)$ : Continuation of Top by lowering the cycle time proportionally. $\tau_{h}, \tau_{c h}=\tau_{h c}, \tau_{c}=$ are: magenta (c) as on Top, 0.0001125, 0.1165, 0.008205 green (d), $0.00005625,0.05825,0.0041025$ maroon (e) The parameters for all the cycles are: $J=1.25, T_{h}=$ $4, T_{c}=3.6, \omega_{h}=11, \omega_{c}=6.5, \kappa_{h}^{\downarrow}=0.36, \kappa_{c}^{\downarrow}=0.0656$, see Eq. (29) 


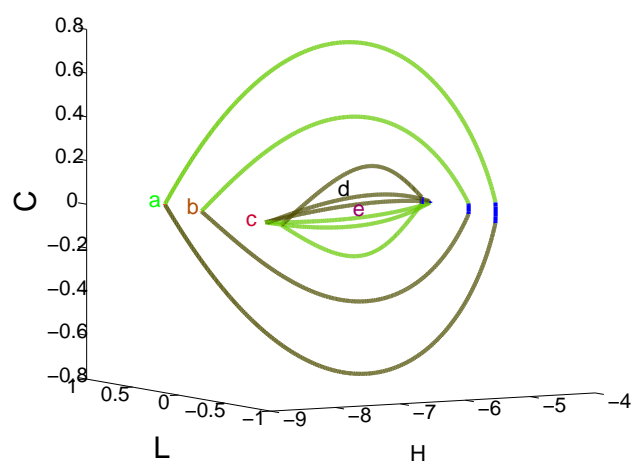

FIG. 8: (Color online)The trajectories in the space $H, L, C$ of the cycles of Fig. 7 where the cycles are indicated by letters corresponding to the cycles of Fig. 7.

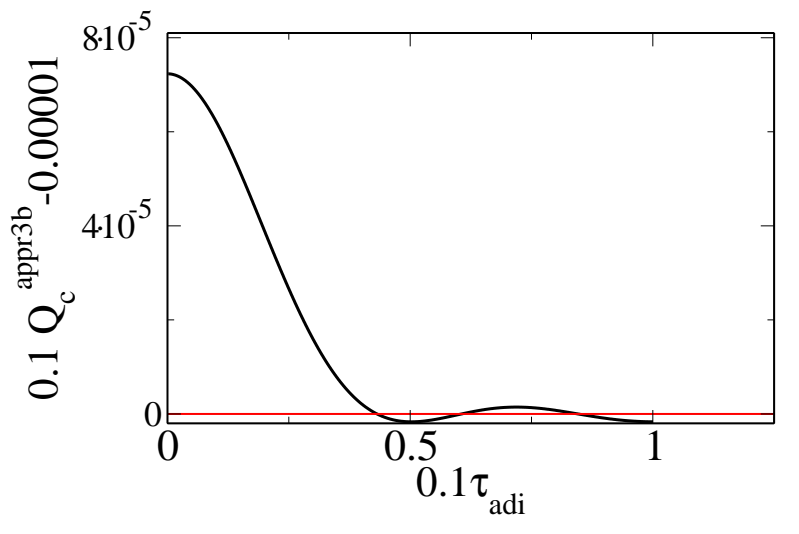

FIG. 9: (Color online) $Q_{c}$ as a function of $\tau_{a d i}$ with some internal scaling as denoted on the figure in order to achieve the exact results by the approximation of Eq. (29), which approximate the cycles of Fig. 7, with the same parameters. The fineness of the sign change is obvious.

The entropy generation $\left(\mathcal{S}^{u}\right)$ for a cyclic process is generated in the baths:

$$
\mathcal{S}^{u} \approx-\left(\frac{\mathcal{Q}_{c}}{T_{c}}+\frac{\mathcal{Q}_{h}}{T_{h}}\right)
$$

An explicit approximations for $\mathbf{C O P}$ and $\mathcal{S}^{u}$ for the case of Eq. (26) is now evaluated. This requires to compute the work input, $\mathcal{W}^{\text {on }}$. Using the notation of Fig. 2,

$$
\mathcal{W}^{o n} \approx\left(E_{C}-E_{D}\right)-\mathcal{Q}_{c}^{a p p r 1 b}
$$




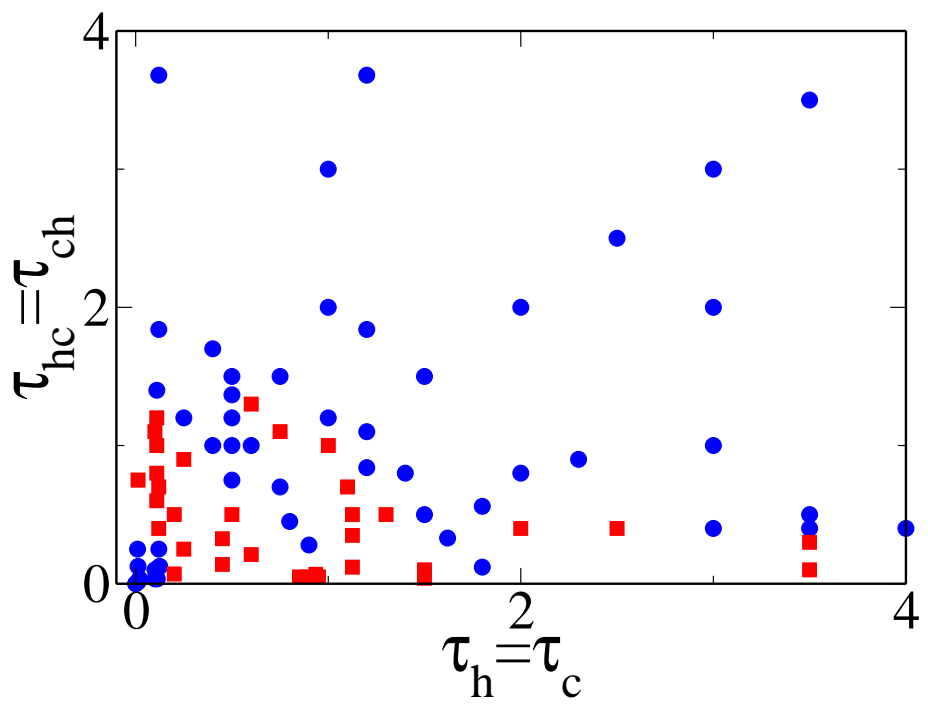

FIG. 10: (Color online)A map of parameter regions of time allocation leading to refrigeration in blue cycles and non refrigeration regions in red squares. The cycle of Fig. 1 is located in the blue island in the lower left corner. The general parameters are: $J=2 ., T_{c}=14, T_{h}=15, \omega_{c}=0.1, \omega_{h}=6$, $\kappa_{h}^{\downarrow}=0.36, \kappa_{c}^{\downarrow}=0.328$. The parameters used fit case 1: Eq. (26).

Similarly to Eq. (111), the values of $E_{C}$ and $E_{D}$ are evaluated from the limit cycle invariant vector $\vec{X}$ of the cycle propagator at points $C$ and $D$.

Denoting again $e^{-\left(\Gamma_{c} \tau_{c}\right)}=\alpha$ and $\cos \left(\Omega_{c} \tau_{c}\right)=c c, \sin \left(\Omega_{c} \tau_{c}\right)=s s$, the work done on the cycle $\left(\mathcal{W}^{o n}\right)$ becomes: $\mathcal{W}^{o n} \approx \frac{\tau_{h} E_{h}^{e q} \Gamma_{h}(1-\alpha)\left(\alpha^{2}-2 \alpha c c+1\right)+\tau_{h}^{2}\left(E_{h}^{e q} \Gamma_{h}^{2} \alpha^{2} s s^{2}(2 \alpha-1)-\frac{\Omega_{h}^{2}}{\Omega_{c}} s s \Gamma_{h} E_{c}^{e q} \alpha(1-\alpha)\right)}{(\alpha-1)\left(\alpha^{2}-2 \alpha c c+1\right)+\Omega_{h}^{2} \tau_{h}^{2} \alpha^{2}(\alpha-c c)-\alpha^{2} \Gamma_{h} \tau_{h}\left(\alpha-2 \alpha \Gamma_{h} \tau_{h}+2 \Gamma_{h} \tau_{h} c c\right)}-\mathcal{Q}_{c}$

when terms with third and larger orders of $\tau_{h}$ are neglected. Noticing that the lowest order of $\tau_{h}$ in the expressions for $\mathcal{Q}_{c}$ were second order. The invested work becomes:

$$
\mathcal{W}^{o n} \approx-\tau_{h} E_{h}^{e q} \Gamma_{h}
$$

Eq. (34) shows that the invested work is on the cold $\rightarrow$ hot adiabat and is dissipated almost exclusivly on the hot isomagnetic segment. 


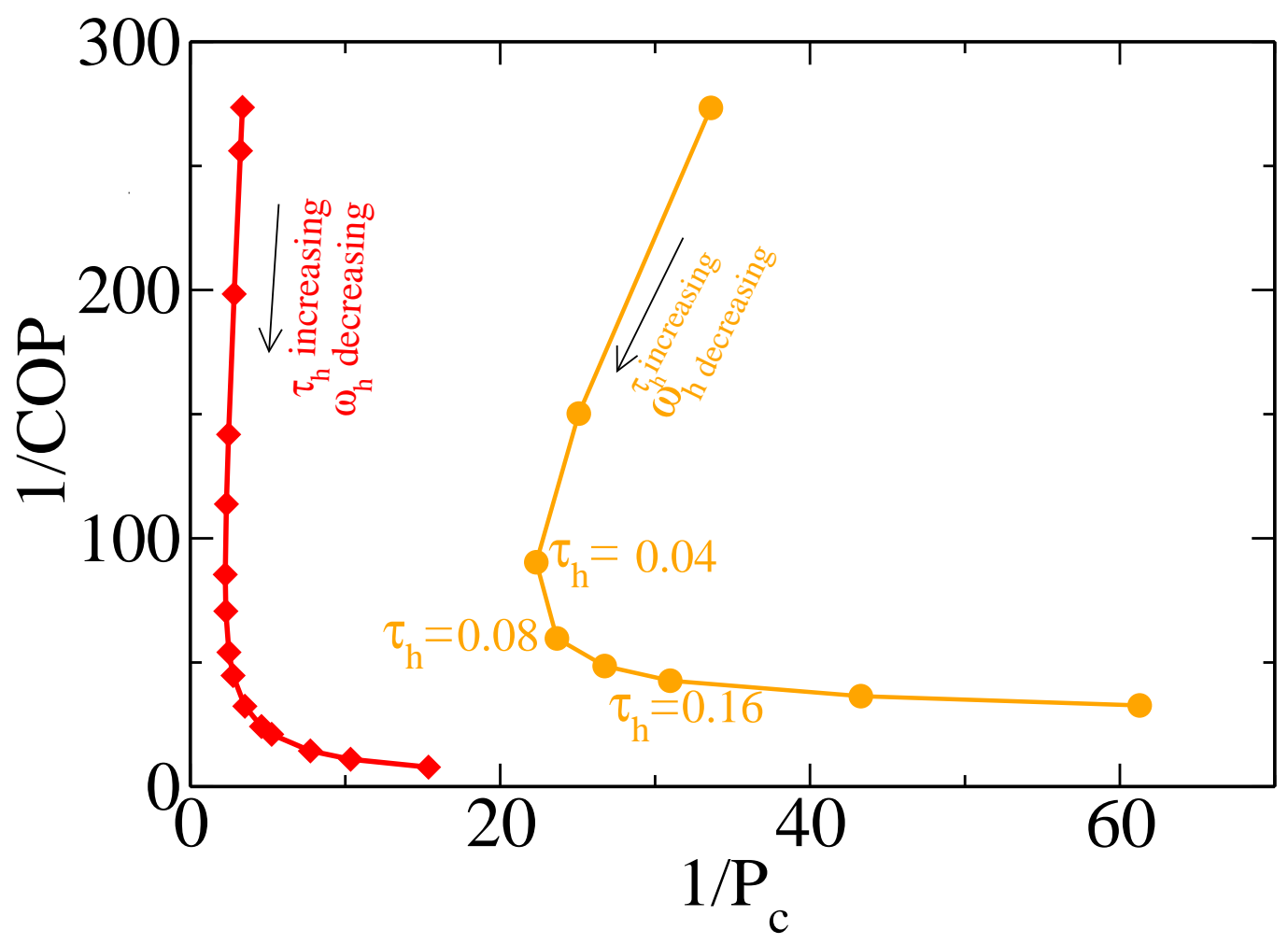

FIG. 11: (Color online) The $\mathbf{1} / \mathbf{C O P}$ as a function of inverse cooling power, $1 / \mathcal{P}_{c}$. The data correspond to the conditions of approximation $Q_{c}^{a p p r 2}$, Eq. (27). The data for both figures are: $J=2.5, T_{h}=10, T_{c}=9, \omega_{c}=2.5, \tau_{c}=0.008375, \tau_{c h}=\tau_{h c}=0.065625$ and also the condition $\left(\omega_{h} \tau_{h}=6.252\right)$. Besides that $\omega_{h}=625.2$ is the largest $\omega_{h}$ value for both plots. The differences between the plots are the $\kappa$ values. For the orange circle figure $\kappa_{c}^{\downarrow}=0.328, \kappa_{h}^{\downarrow}=0.36$, where three points correspond to the three cycles of Fig. 5, whose $\tau_{h}$ values are denoted on the figure. The largest $\tau_{h}$ is 0.32 . For the red diamond plot $\kappa_{h}^{\downarrow}=3.6$, and $\kappa_{c}^{\downarrow}=3.28$. For this figure the largest $\tau_{h}$ is 0.5157905 .

The COP of the cycle is approximated as:

$$
\mathrm{COP} \approx \tau_{\mathrm{h}} \frac{\alpha\left(\Omega_{\mathrm{c}} \mathrm{ssE}_{\mathrm{h}}^{\mathrm{eq}} \Gamma_{\mathrm{h}}-\Omega_{\mathrm{h}}^{2} \mathrm{E}_{\mathrm{c}}^{\mathrm{eq}} \mathrm{cc}+\mathrm{E}_{\mathrm{c}}^{\mathrm{eq}} \alpha \Omega_{\mathrm{h}}^{2}\right)}{\mathrm{E}_{\mathrm{h}}^{\mathrm{eq}} \Gamma_{\mathrm{h}}\left(\alpha^{2}-2 \alpha \mathrm{cc}+1\right)}
$$

To compute the approximation for $\mathcal{S}^{u}$, according to Eq. (31), we use $\mathcal{Q}_{C}$ from Eq. (26). $\mathcal{Q}_{h}$ requires an additional approximation. To first order $\mathcal{Q}_{h}=-\mathcal{W}^{o n}$, when $\mathcal{W}^{\text {on }}$ is given 


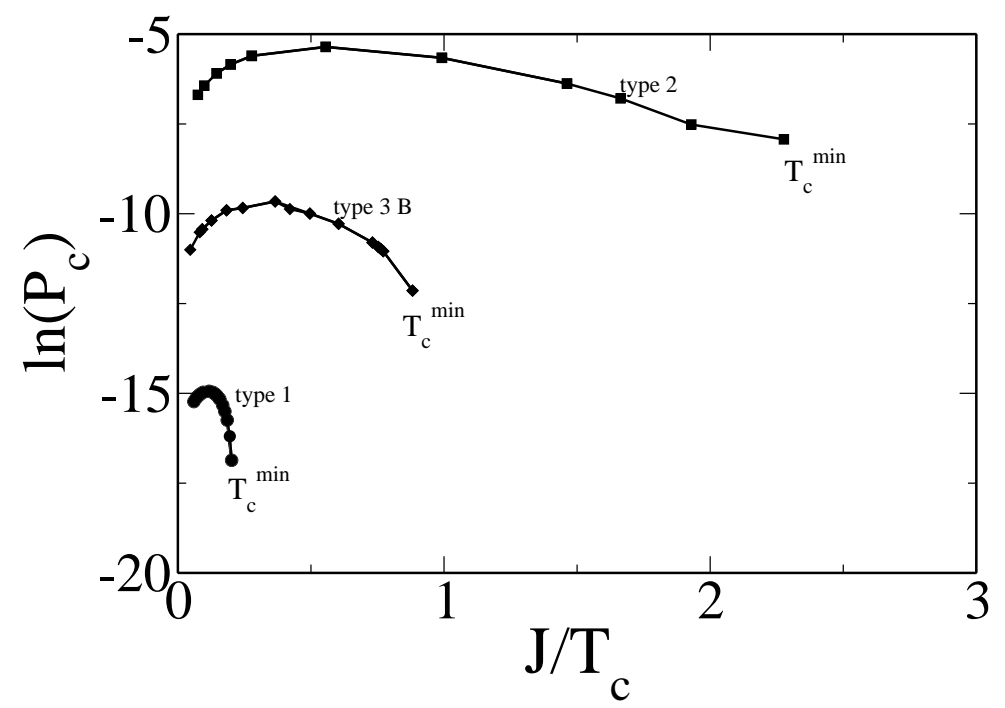

FIG. 12: $\ln \left(\mathcal{P}_{c}\right)$ as a function of $J / T_{c}$ for the three classes of sudden refrigerators. The cycle parameters for type 1 correspond to Fig. 1 where $T_{c} / T_{h}$ was kept constant and $T_{c}$ was varied. The cycle parameters of type 2 correspond to Fig. 2 where $T_{c} / T_{h}$ was kept constant and $T_{c}$ was varied. The cycle parameters of type $3 \mathrm{~b}$ correspond to Fig. 7 where $T_{c} / T_{h}$ was kept constant and $T_{c}$ was varied.

by Eq. (34). therefore $\mathcal{S}^{u}$ becomes:

$$
\mathcal{S}^{u} \approx-\left(\frac{\alpha \tau_{h}^{2}\left(\Omega_{c} s s E_{h}^{e q} \Gamma_{h}-\Omega_{h}^{2} E_{c}^{e q} c c+E_{c}^{e q} \alpha \Omega_{h}^{2}\right)}{\left(\alpha^{2}-2 \alpha c c+1\right) T_{c}}+\frac{\tau_{h} E_{h}^{e q} \Gamma_{h}}{T_{h}}\right) \approx-\frac{\tau_{h} E_{h}^{e q} \Gamma_{h}}{T_{h}}
$$

Eq. (36) shows that most of the entropy production is generated on the hot isomagnetic segment. Fig. 11 present the dependence of the $\mathbf{1} / \mathbf{C O P}$ on $1 / \mathcal{P}_{c}$, for the subfamily of cycles of Eq. (27). The cycles are chosen with the condition $\omega_{h} \tau_{h}=$ constant, for two different heat transport coefficients to the bath. This analysis in inspired by the studies of Gordon et al. [34, 35]. where a universal behavior of optimal cycles with preassigned cycle times was observed. For the sudden cycles a continuous behavior is only local, nevertheless for the particular family by choosing $\omega_{h} \tau_{h}=$ constant we found a similar behavior.

\section{THE INFLUENCE OF COOLING}

The cooling power $\mathcal{P}_{c}=\mathcal{Q}_{c} / \tau$ as a function of the cold bath temperature $J / T_{c}$, is shown in Fig. 12, A similar plot for the regular cycles can be found in ref. [29]. Common 
to both cases is a minimum temperature beyond which the cycle cannot cool any longer. This minimum temperature is obtained when the occupation of the exited level after the demagnetization segment is larger than the equilibrium value at the cold bath. When $T_{c}$ is increased the cooling power increases exponentially with the equilibrium occupation at $T_{c}$. This minimum temperature is different for the different classes of sudden refrigerators where type 2 outperforms the other classes in both cooling power and minimum temperature. In addition a maximum cooling rate at $T_{c}>J$ is observed, for all the three classes. This is in contrast to a monotonic decrease in $\mathcal{P}_{c}$ in the regular cycles [22, 29]. The maximum can be attributed to the inability to dissipate at the hot bath in a very short time the heat at the hot bath. This is a characteristic of the global behavior of the sudden cycles.

The maximum in the cooling rate is also reflected in the approximation Eq. (26) where $\Omega_{c} \sim J$. Therefore there exists a positive root of $x=\left(J / 2 T_{c}\right)$ in the equation:

$$
\frac{\partial \ln \left(\mathcal{Q}_{c}^{\text {appr } 1 b} / \tau\right)}{\partial\left(J / 2 T_{c}\right)} \equiv \frac{1}{\mathcal{Q}_{c}^{a p p r 1 b}} \frac{\partial\left(\mathcal{Q}_{c}^{\text {appr } 1 b}\right)}{\partial\left(J / 2 T_{c}\right)}=0
$$

The derivation is constrained by the fact that as the temperature of the cold bath is varied, also proportionally the temperature of the hot bath changes. Writing $T_{c} / T_{h}=C^{T}$, therefore not only $E_{c}^{e q}$ and $\Gamma_{c}$ are dependent on $T_{c}$, but also $E_{h}^{e q}$ and $\Gamma_{h}$. After quite tedious computation, neglecting terms by order of magnitude considerations, and taking into account that in the case $\Omega_{c} \sim J$ one obtains:

$$
\frac{1}{\mathcal{Q}_{c}^{a p p r 1 b}} \frac{\partial\left(\mathcal{Q}_{c}^{a p p r 1 b}\right)}{\partial\left(J / 2 T_{c}\right)}=2 \kappa_{c}^{\downarrow} \tau_{c} \exp ^{-2 x}+\frac{1}{2 J \sinh (2 x)}-4 \kappa_{c}^{\downarrow} \tau_{c} \exp ^{-2 x}=0
$$

which leads to:

$$
\left(\frac{J}{2 T_{c}}\right)^{\max }=-\frac{1}{4} \ln \left(1-\frac{1}{2 J \kappa_{c}^{\downarrow} \tau_{c}}\right)
$$

The maximum in Eq. (39) is quite delicate, this comes about since the constraints on the parameters are very restrictive. In addition Eq. (39) is independent of the ratio $C^{T}$, as well as other parameters of the hot segment of the cycle. On the other hand, $\left(J / 2 T_{c}\right)^{\max }$ depends on all the parameters of the cold segment. This suggests that the the maximum point of $\mathcal{P}_{c}$ could be found also for the case where the hot bath has constant temperature.

\section{DISCUSSION}

One can say, that practicing science involves making order in seemingly disorder. The ensemble of the sudden cycles is an extreme example of that statement. For example, we 
saw both in Fig (10) and Fig (77) that changing the time allocations continuously results with a large number of discontinuities. Therefore continuity, one of the main aids in research, doesn't help in the case of sudden cycles. Also, when one changes slightly the bath temperatures, or transition probabilities, or the field values might result with $P_{c}<0$. One can try to optimize $P_{c}$ as a function of time allocation, a standard procedure for regular cycles, generally doesn't work for sudden cycles. The result in most cases will be, that while continuously changing the time allocations - a jump will occur from refrigerator to non refrigerator.

The refrigerators studied belong to the family of four stroke Otto refrigerators [9, 19, 22, 24]. The working fluid composed from an ensemble of spin pairs, which is a simplified model of a working medium composed of magnetic salts. For this model the dynamics is described by the equation of motion for the thermodynamical observables. The present study focuses on refrigerators with cycle times shorter or much shorter than the internal time scale of the working fluid. As a result the different segments of the Otto cycle become interconnected. This characteristic results in the density operator $\hat{\boldsymbol{\rho}}$ deviating from the typical diagonal form in the energy representation. As a consequence, the energy entropy was always much larger than the Von Neumann entropy. These cycles termed sudden cycles, settle to a limit cycle, typically after a large number of iterations. The state of this limit cycle is the eigenvector of the cycle's global propagator with eigenvalue one. This property is exploited to study the performance of the cycles using a vector space of a closed set of operators which is sufficient to represent the density operator of the limit cycle.

The total cycle is analyzed through segment propagators which map the vector space of operators. Our classification scheme of families of sudden cycles is based on analytical approximate expressions for the propagators on each segment. These segment propagators were then synthesized to global propagators. Having done that, we computed the approximate eigenvectors with an eigenvalue one for each limit cycle. These approximate solution demonstrate the global property of the cycle. We find a special continuous subset of cycles whose behavior is similar to the universal plot of Gordon et al. [34, 35]. It was shown in the study that the entropy production $\mathcal{S}^{u}$ is generated on the boundary of the hot bath. Finally, the sudden cycles possess a maximum cooling power as a function of $J / T_{c}$ as opposed to the regular cycles where we demonstrated a monotonic decrease of $\mathcal{P}_{c}$.

Finite time thermodynamics has been devoted to the study of systems far from equilib- 
rium. For example the energy distribution of the working medium was not in the thermal Gibbs state. The present study is characterized by coherence, a deviation from a Gibbs state because the state does not commute with the stationary Hamiltonian.

\section{Acknowledgments}

The study was supported by the Israel Science Foundation. We thank Amikam Levi, Yair Rezek, Gershon Kuritzky, Robert Alicki and David Gelbwaser for stimulating discussions.

\section{Appendix A: Thermodynamical Relations.}

In order to fulfill the second law of thermodynamics, the maximal efficiency, $\eta^{\max }$, of a heat engine with working fluid of two coupled spins(with the corresponding Carnot efficiency relation) is Cf. [10]:

$$
\eta^{\max }=1-\frac{\Omega_{c}}{\Omega_{h}}<1-\frac{\omega_{c}}{\omega_{h}}<1-\frac{T_{c}}{T_{h}}
$$

For the reverse operation as a refrigerator with the same working fluid, the basic inequality must change its direction, and as a consequence from Eq. A1, the constraint on the minimum cold bath temperature $T_{c}$ will be

$$
\begin{gathered}
T_{c} \geq \frac{\Omega_{c}}{\Omega_{h}} T_{h} \geq \frac{J}{\Omega_{h}} T_{h} \\
\operatorname{COP}^{\text {Carnot }}=\frac{T_{c}}{T_{h}-T_{c}} ; \operatorname{COP}^{\text {Otto }}=\frac{\Omega_{c}}{\Omega_{h}-\Omega_{c}}
\end{gathered}
$$


[1] W J Haas, E C Wiersma, H A Kramers, Physica [1], 1 (1934).

[2] W A Wolf, Phys. Rev. 115, 1196 (1959).

[3] P Hakonen, O V Lounasmaa and A Oja, J. Magn. Magn. Mat. 100, 394 (1991).

[4] K A Gschneider Jr, V K Pecharsky and A O Tsokol, Rep. on Prog. in Phys. 68, 1479 (2005).

[5] Feng Wu, Lingen Chen, Shuang Wu and Fengrui Sun, J. Phys D: Applied Phys. 39, 4731 (2006).

[6] M Fattori, T Koch, S Goetz, A Griesmaier, S Hensler, J Stuhler and T Pfau, Nature Physics [2], 765 (2006).

[7] E. G. R. Kosloff and J. M. Gordon, J. App. Phys. 87, 8093 (2000).

[8] R. K. T. Feldmann, E. Geva and P. Salamon, Am. J. Phys. 64, 485 (1996).

[9] T. Feldmann and R. Kosloff, Phys. Rev. E 61, 4774 (2000).

[10] T. Feldmann and R. Kosloff, Phys. Rev. E 68, 016101 (2003).

[11] T. Feldmann and R. Kosloff, Phys. Rev. E 73, 025107(R) (2006).

[12] A Rowe and a Tura, Int. J. of Refrigeration 29, 1286 (2006).

[13] S Hensler, A Greiner, J Stuhler and T Pfau, Eur. Phys. Lett. 71, 918 (2005).

[14] Y. Rezek, R. Kosloff, New Jour. of Phys. 8(83), 1 (2006).

[15] D. Segal and A. Nitzan, Phys. Rev. E 73, 026109 (2006).

[16] E. Geva and R. Kosloff, J. Chem. Phys. 96, 3054 (1992).

[17] He JiZhou, He Xian and Tang Wei, Science in China Series G-Phys. Mech. \& Ast. 52, 1317 (2009).

[18] H T Quan, Yu-xi Liu, C P Sun and Franco Nori, Phys. Rev. E 76, 031105 (2007).

[19] Y Rezek, P Salamon, K H Hoffmann and R Kosloff, Eur. Phys. Lett. 85, 30008 (2009).

[20] A. E. Allahverdyan, K. Hovhannisyan, and G. Mahler, Phys. Rev. E 81, 051129 (2010).

[21] N. Linden, S. Popescu, P. Skrzypczyk, Phys.Rev.Lett. 105, 130401 (2010).

[22] Tova Feldmann and Ronnie Kosloff, Eur. Phys. Lett. 89, 20004 (2010).

[23] A. E. Allahverdyan, K. V. Hovhannisyan, D. Janzing, and G. Mahler, Phys. Rev. E 84, 041109 (2011).

[24] K. H. Hoffmann, P. Salamon, Y. Rezek and R. Kosloff, Eur. Phys. Lett. 96, 60015 (2011).

[25] R. Kosloff and T. Feldmann, Phys. Rev. E 65, 055102 (2002). 
[26] G. Thomas and R. S. Johal, Phys. Rev. E 83, 031135 (2011).

[27] K. Kraus, Ann.Phys. 64, 311 (1971).

[28] T. Feldmann and R. Kosloff, Phys. Rev. E 70, 046110 (2004).

[29] Ronnie Kosloff and Tova Feldmann, Phys. Rev. E 82, 011134 (2010).

[30] Xi Chen, A. Ruschhaupt, S. Schmidt, A. del Campo, D. Guery-Odelin, J. G. Muga, Phys.Rev.Lett. 104, 063002 (2010).

[31] G. Lindblad, Comm. Math. Phys. 48, 119 (1976).

[32] R. Alicki and K. Lendi, Quantum Dynamical Semigroups and Applications (Springer-Verlag, Berlin, 1987).

[33] Uri Banin, Allon Bartana, Sanford Ruhman and Ronnie Kosloff, J. Chem. Phys. 101, 8461 (1994).

[34] J. M. Gordon and K. C. Ng, J. App. Phys. 75, 2769 (1994).

[35] J M Gordon, K C Ng and H.T. Chua, Int. J. of Refrigeration 20, 191 (1997). 